\title{
Erratum: Computation of the Correlated Metal-Insulator Transition in Vanadium Dioxide from First Principles [Phys. Rev. Lett. 114, 176401 (2015)]
}

\author{
Huihuo Zheng and Lucas K. Wagner
}

(Received 19 December 2017; published 31 January 2018)

DOI: 10.1103/PhysRevLett.120.059901

We predicted that the insulating state in $\mathrm{VO}_{2}$ would have a singlet-triplet excitation around 123(6) meV. Recently, inelastic x-ray scattering measurements [1] found this excitation at $460 \mathrm{meV}$. It has been noted that this result is far from the quantum Monte Carlo result we obtained [1,2]. On reanalysis of our original computational data, we noticed two errors that bring the results in closer alignment. The first error was due to a confusion of formula units and crystallographic units, and the second has to do with the interpretation of the calculation. The corrected value of the singlet-triplet gap from our quantum Monte Carlo calculation is $440(24) \mathrm{meV}$, which agrees with the experimental result within statistical error.

We computed the frozen spin states of the dimers, $|\uparrow \uparrow\rangle$ and $|\uparrow \downarrow\rangle$. For a dimer Heisenberg model,

$$
H=J S_{1} \cdot S_{2}=\frac{1}{2} J\left[\left(S_{1}+S_{2}\right)^{2}-S_{2}^{2}-S_{1}^{2}\right]=\frac{1}{2} J[s(s+1)-3 / 2] .
$$

For the two frozen spin states,

$$
E(\uparrow \uparrow)=\left\langle\uparrow \uparrow\left|J S_{1} \cdot S_{2}\right| \uparrow \uparrow\right\rangle=J / 4
$$

and

$$
E(\uparrow \downarrow)=\left\langle\uparrow \downarrow\left|J S_{1} \cdot S_{2}\right| \uparrow \downarrow\right\rangle=-J / 4 .
$$

The energy difference between these two states is thus $J / 2$. The singlet eigenstate $\sqrt{1 / 2}(|\uparrow \downarrow\rangle-|\downarrow \uparrow\rangle$ ) (with $s=0$ ) has energy $-3 J / 4$ while the triplet eigenstate $|\uparrow \uparrow\rangle$ (with $s=1$ ) has energy $J / 4$, so the singlet-triplet excitation energy is $J$.

We computed using quantum Monte Carlo calculations the following difference of energy expectation values for $\mathrm{VO}_{2}$ in the insulating state:

$$
E(\uparrow \uparrow)-E(\uparrow \downarrow)=123(6) \mathrm{meV} / \mathrm{VO}_{2} .
$$

The first error is that we should have computed energy per dimer, which is twice as large. The corrected equation for a dimer is thus

$$
E(\uparrow \uparrow)-E(\uparrow \downarrow)=246(12) \mathrm{meV} / \operatorname{dimer}=J / 2 .
$$

Our second error was setting $E(\uparrow \uparrow)-E(\uparrow \downarrow)$ as the singlet-triplet gap, which incorrectly ignored spin fluctuations as noted in the previous paragraph. Therefore, the singlet-triplet excitation is $J=492(24) \mathrm{meV}$, within two statistical error bars of the experimental result. This realization was made possible by work we have done regarding effective model fitting $[3,4]$. We view this as a strong motivation to have a rigorous theory of effective models as we tried to lay out in those papers.

The above estimation of $J$ ignores the weak interdimer coupling. One can include interdimer couplings using our calculations as well. In that case, the spin interaction is

$$
\mathcal{H}=\sum_{i} J_{\text {intra }} S_{i} \cdot S_{i+1}+J_{\text {inter }} S_{i+1} \cdot S_{i+2}+E_{0}
$$


where $S_{i}=\frac{1}{2} \sigma_{i}$ and the $\sigma_{i}$ 's are Pauli matrices. In our quantum Monte Carlo simulation, we calculated the energy of three frozen orders, the ferromagnetic order $(\uparrow \uparrow \uparrow \uparrow \ldots)$, antiferromagnetic order $(\uparrow \downarrow \uparrow \downarrow \ldots)$, and intra-antiferromagnetic order $(\uparrow \downarrow \downarrow \uparrow \ldots)$,

$$
\begin{gathered}
E_{\mathrm{AFM}}=-\frac{N}{8}\left(J_{\text {intra }}+J_{\text {inter }}\right)+E_{0}, \\
E_{\mathrm{FM}}=\frac{N}{8}\left(J_{\text {intra }}+J_{\text {inter }}\right)+E_{0}, \\
E_{\text {intraAFM }}=-\frac{N}{8}\left(J_{\text {intra }}-J_{\text {inter }}\right)+E_{0},
\end{gathered}
$$

where $N$ is the number of $\mathrm{VO}_{2}$ formula units in the system. From Eq. (7) we obtain $J_{\text {intra }}=4\left(E_{\mathrm{FM}}-E_{\text {intraAFM }}\right) /$ $N=440(24) \mathrm{meV}$, and $J_{\text {inter }}=4\left(E_{\text {intraAFM }}-E_{\mathrm{AFM}}\right) / N=52(24) \mathrm{meV}$ [5]. The singlet-triplet excitation energy $J_{\text {intra }}$ is 440 (24) $\mathrm{meV}$. This is slightly closer to the experimental value, but is a relatively small correction to the above two-site only model considering the statistical uncertainties.

In this case, the quantum Monte Carlo calculations helped to make it clear that indeed there should be a singlet-triplet excitation, and we hope that the experiments were partially motivated by the work to search for it. As a way of understanding the ground state of $\mathrm{VO}_{2}$, we view this as a resounding success. The quantum Monte Carlo results themselves were extremely accurate; it was our interpretation of the computational results that resulted in an incorrect quantitative prediction.

[1] H. He, A. X. Gray, P. Granitzka, J. W. Jeong, N. P. Aetukuri, R. Kukreja, L. Miao, S. A. Breitweiser, J. Wu, Y. B. Huang et al., Measurement of collective excitations in $\mathrm{VO}_{2}$ by resonant inelastic x-ray scattering, Phys. Rev. B 94, 161119 (2016).

[2] W. H. Brito, M. C. O. Aguiar, K. Haule, and G. Kotliar, Dynamic electronic correlation effects in $\mathrm{NbO}_{2}$ as compared to $\mathrm{VO}_{2}$, Phys. Rev. B 96, 195102 (2017).

[3] H. J. Changlani, H. Zheng, and L. K. Wagner, Density-matrix based determination of low-energy model Hamiltonians from ab initio wavefunctions, J. Chem. Phys. 143, 102814 (2015).

[4] H. Zheng, H. J. Changlani, K. T. Williams, B. Busemeyer, and L. K. Wagner, From real materials to model Hamiltonians with density matrix downfolding, arXiv:1712.00477.

[5] Based on our quantum Monte Carlo results, $\left(E_{\mathrm{FM}}-E_{\mathrm{AFM}}\right) / N=123(6) \mathrm{meV},\left(E_{\text {intraAFM }}-E_{\mathrm{AFM}}\right) / N=13(6) \mathrm{meV}$. 